\section{Development of a Disease-} Modifying OA Drug (DMOAD) in Knee Osteoarthritis: The Example of Sprifermin

\author{
K. Marhardt ${ }^{1}$, N. Muurahainen ${ }^{2}$ \\ ${ }^{1}$ Merck Gesellschaft mbH, Vienna, Austria* \\ ${ }^{2}$ EMD Serono, Rockland/MA, USA*
}

1. Introduction: $\mathrm{OA}$ and unmet clinical need for a DMOAD

2. Past DMOAD drug development

3. Sprifermin, its MOA, and clinical development program

4. Lessons learned, summary, and next steps

It is estimated that osteoarthritis (OA) affects 150 million people around the world. While OA is the most common cause of physical disability in older adults, the average age at diagnosis is around 55 years. Currently available drugs to treat OA primarily provide symptom relief. Accordingly, there is a strong unmet need for a Disease-Modifying OA Drug (DMOAD), i.e., a drug that will alter OA disease progression by improving joint structure and ameliorating symptoms, either by reducing pain or improving physical function.

In the past, a number of potential DMOADS were investigated - including three compounds recently investigated in large Phase III clinical development programs, i.e. cindunistat, strontium ranelate, and oral salmon calcitonin (Hellio Le Graverand 2012, Reginster 2012, and Karsdal 2014). Some of these trials measured structure on MRI in subsets of subjects, but none of these obtained MRI results in all subjects. Some drugs showed trends toward improvement on X-ray, but efficacy effects were not statistically signficant (Hellio Le Graverand 2012, Brandt 2005). Atlhough there have been many clinical development programs of drugs to treat $\mathrm{OA}$, no DMOAD has yet been approved by European Union regulatory bodies or FDA.

Sprifermin, a truncated form of fibroblast growth factor 18 (rhFGF18), is currently being investigated as a potential DMOAD. Sprifermin induces cartilage formation by increasing chondrocyte proliferation, resulting in increased overall extracellular matrix production by chondrocytes.

Two clinical trials of intra-articularly (i.a.) injected sprifermin vs. placebo have been completed: (1) a Phase 1 Firstin-Human (FiH) trial (clinicaltrials.gov NCT00911469) and (2) a Phase 1b trial (NCT01033994). Both trials found no major safety or injection-site issues on sprifermin.

The FiH trial is a randomized double-blind placebo-controlled (DBPC) trial that was performed in patients with severe knee $\mathrm{OA}$ who were scheduled for total knee replacement (TKR). It evaluated the safety of intra-articular sprifermin (primary); systemic exposure, biomarkers, and explored cartilage histology obtained at the time of TKR.

The Phase $1 \mathrm{~b}$ trial is a DBPC trial performed in patients with moderate knee OA (Kellgren-Lawrence Grade [KLG] 2-3) who were not scheduled for TKR. It included many endpoints, in- cluding: cartilage thickness measured by magnetic resonance imaging (MRI) (primary), joint space width by X-ray, symptoms on the Western Ontario McMaster (WOMAC) OA index, and biomarkers. In this trial, dose-dependent reductions in structural changes were observed on sprifermin as compared to placebo. The WOMAC pain score improved in all patients. Lessons learned: One challenge to designing clinical trials of i. a. DMOADs is the known pain-reducing effect of i.a. placebo. A challenge to the development of all DMOADs in general is that the effects of the drug on multiple joint structures are observed on MRI, but not on X-ray. Another challenge to developing DMOADs in general is that $\mathrm{OA}$ is a heterogeneous disease. No one DMOAD may be able to treat all sub-types of $\mathrm{OA}$. In the future, ideally biomarkers may aid in the identification of different $\mathrm{OA}$ responder sub-types.

Summary and next steps: DMOAD development is challenging. Additional clinical trials are needed to replicate the positive results from early sprifermin trials and to confirm optimal dosing. A Phase II dose-ranging DBPC trial of sprifermin is currently ongoing (NCT01919164). It will assess structure in all patients both by MRI and X-ray, evaluate symptoms via WOMAC and other patient-reported outcome mesasures, while also exploring the potential for future biomarker prediction of subjects who are clinical responders to treatment. Interessenkonflikt: Dr. Marhardt ist Mitarbeiter der Firma Merck Gesellschaft mbH und Dr. Muurahainen ist Mitarbeiterin der Firma EMD Serono.

\section{References}

1 Hellio Le Graverand MP, Clemmer RS, Redifer P et al. A 2-year randomised, double-blind, placebo-controlled, multicentre study of oral selective iNOS inhibitor, cindunistat (SD-6010), in patients with symptomatic osteoarthritis of the knee. Ann Rheum Dis 2013; 72: 187-195

2 Reginster JY, Badurski J, Bellamy N et al. Efficacy and safety of strontium ranelate in the treatment of knee osteoarthritis: results of a doubleblind, randomised placebo-controlled trial. Ann Rheum Dis 2013; 72: 179-186

3 Karsdal MA, Byrjalsen I, Alexandersen P et al. Treatment of symptomatic knee osteoarthritis with oral salmon calcitonin: results from two phase 3 trials. Osteoarthritis Cartilage 2015; 23: 532-543

4 Brandt KD, Mazzuca SA. Lessons learned from nine clinical trails of disease modifying osteoarthritis drugs. Arthritis \& Rheumatism 2005; 52: $3349-3359$

Bibliography

Dol http://dx.doi.org/10.1055/s-0035-1558063

Drug Res 2015; 65, Suppl. 1: S13-S13

(c) Georg Thieme Verlag KG Stuttgart · New York .

ISSN 2194-9379

\section{Correspondence \\ Dr. Kurt Marhardt}

Merck Gesellschaft mbH

Zimbagasse 5

1147 Wien

Österreich

kurt.marhardt@merckgroup.com

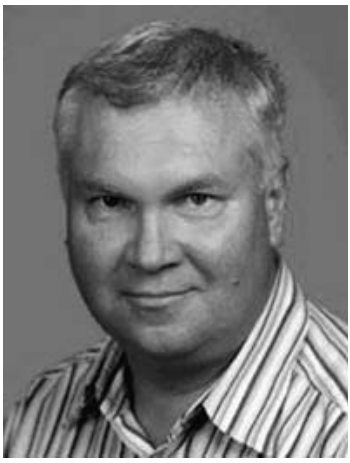

